\title{
New boundary conditions for fluid interaction with hydrophobic surface
}

\author{
František Pochylý ${ }^{1}$, Simona Fialová ${ }^{1 *}$, and Michal Havlásek ${ }^{1}$ \\ ${ }^{1}$ Brno University of Technology, Faculty of Mechanical Engineering, Victor Kaplan Department of Fluids Engineering, \\ Technická 2896/2, 61669 Brno, Czech Republic
}

\begin{abstract}
Solution of both laminar and turbulent flow with consideration of hydrophobic surface is based on the original Navier assumption that the shear stress on the hydrophobic surface is directly proportional to the slipping velocity. In the previous work a laminar flow analysis with different boundary conditions was performed. The shear stress value on the tube walls directly depends on the pressure gradient. In the solution of the turbulent flow by the k- $\varepsilon$ model, the occurrence of the fluctuation components of velocity on the hydrophobic surface is considered. The fluctuation components of the velocity affect the size of the adhesive forces. We assume that the boundary condition for $\varepsilon$ depending on the velocity gradients will not need to be changed. When the liquid slips over the surface, non-zero fluctuation velocity components occur in the turbulent flow. These determine the non-zero value of the turbulent kinetic energy $K$. In addition, the fluctuation velocity components also influence the value of the adhesive forces, so it is necessary to include these in the formulation of new boundary conditions for turbulent flow on the hydrophobic surface.
\end{abstract}

\section{Introduction}

Solution of both laminar and turbulent flow with consideration of hydrophobic surface is based on the original Navier assumption that the shear stress on the hydrophobic surface is directly proportional to the slipping velocity 1 . This condition is supplemented by Tesk's assumption that the slip will occur after a certain shear stress threshold has been exceeded. To solve more complicated liquid flow tasks over a curved surface 2 , the mentioned boundary condition is supplemented by other terms. Generally on the surface $\mathrm{S}$ for the shear stress vector $\boldsymbol{\tau}$ applies:

$$
c=0:|\tau| \leq|\tau|_{K} \quad c \neq 0: \tau=k c
$$

This boundary condition is also advantageous because it determines the zero normal component of the velocity to the surface that must be met if the boundary is not porous.

In previous work, a laminar flow analysis with different boundary conditions was performed, depending on the shear stress characterizing the slip of the liquid (water in this case) on the hydrophobic surface with the following conclusions:

- The fluid slip on the surface is subject of a low surface energy.

- The surface energy can be judged by the value of the contact angle of the liquid droplet on the surface 8 .

$\Theta<80^{\circ}$ - hydrophilic surface (wettable surface).

$\Theta>80^{\circ}$ - hydrophobic surface (surface is non wettable).

- Surface energy fundamentally affects the shear stress value on the solid-liquid interphase 3 . The shear stress value on the tube walls directly depends on the pressure gradient. At low surface energy the fluid slips on the surface. The hydrophobic surface is characterized by adhesive forces dependent on adhesion coefficient $\alpha$. For $\alpha=100$, the surface already behaves as hydrophilic.

Based on experiments, it has been found that the hydrophobic surface has the ability to bind air molecules $4,5,6$. It retains this property even during the laminar flow of the water in pipes. The air layer adjacent to the surface changes the viscosity in this area 7.

* Corresponding author: fialova@fme.vutbr.cz 


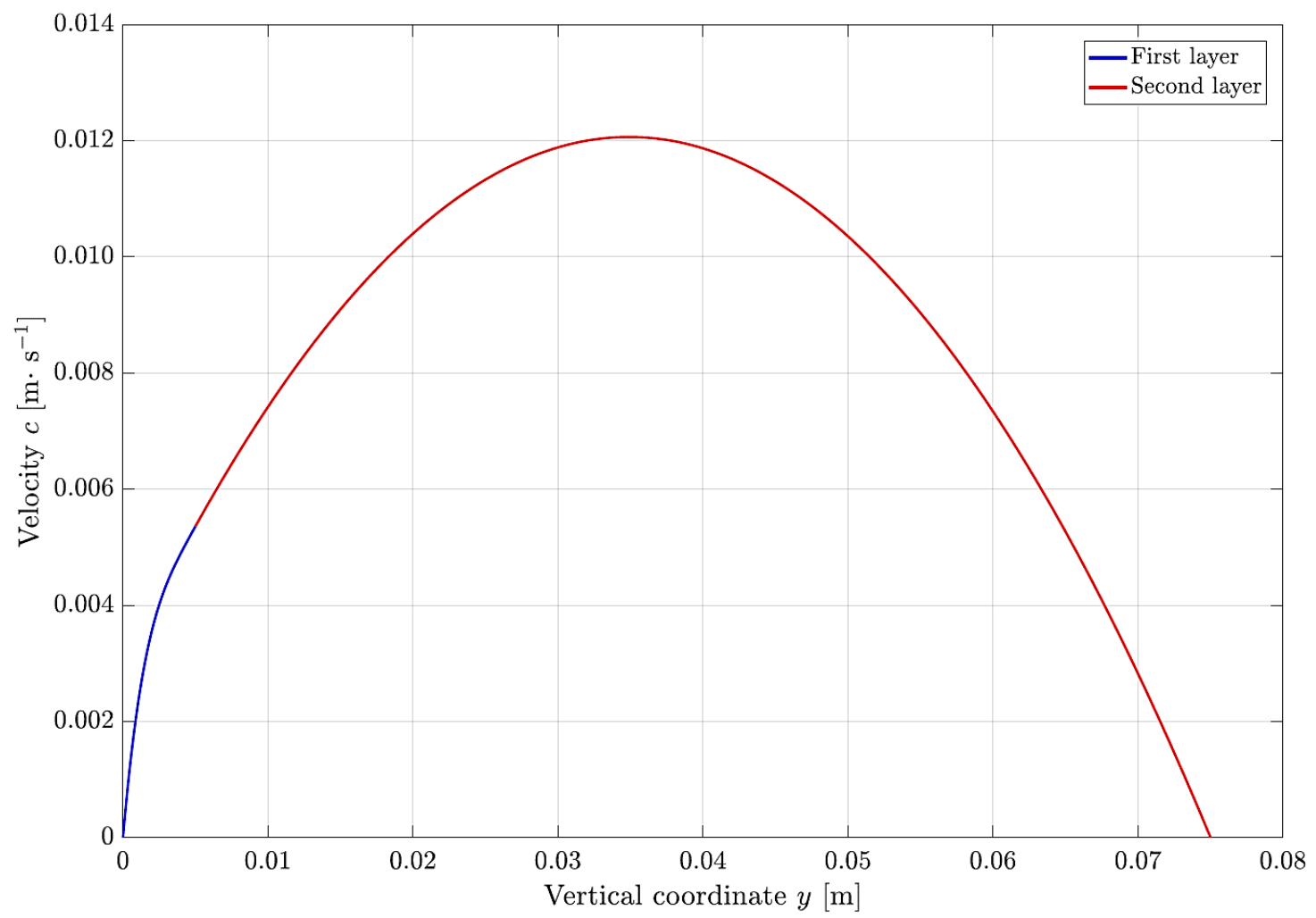

Fig. 1. Velocity profile shape $\Delta \mathrm{p}=0.015 \mathrm{~Pa}, \tau_{0}=0,05379 \tau_{K R}, \chi=3,752457.10^{9} 1 / \mathrm{Pasm}^{2}$

\section{Turbulent Flow}

It is supposed that the viscosity in the interval varies according to:

$$
\eta_{0}=\frac{\eta}{1+\chi \eta(h-y)^{2}}
$$

The viscosity value depends on the constant $\chi$ that represents the degree of saturation by gas:

For $\chi>0, \quad \eta_{0}$ on the surface decreases

For $\chi<0, \quad \eta_{0}$ on the surface increases

this value also depends on the shear stresses on the surface. In terms of qualitative analysis, it is particularly interesting to see Fig.1, which shows the laminar velocity profile of the flow in two layers of different viscosity (on the left side there is $\eta_{0}$ and on the right side $\eta>\eta_{0}$ ).

From Fig. 1, a steep increase of the velocity profile at low viscosity is evident. The velocity profile is characteristic especially for the air layer adhering to the surface of the tube $(c=0)$. When evaluating experiments using the PIV method (which cannot measure the velocity profile near the wall), it could be incorrectly judged from the figure, that the liquid slips along the wall of the tube. This is because we would have the last measured point where the first layer ends (the end of the blue color). That is why certain caution should be applied when evaluating results of the PIV experiments 6 .
The experiment also shows that the thickness of the air layer decreases as the Reynolds number increases and in turbulent flow, at higher Reynolds number values it is completely flushed.

When the air plastron is flushed, the adhesion forces act on the liquid at the surface. Adhesion forces are characterized by the adhesion coefficient $k$ or its nondimensional variant $\gamma$. Coefficient $k$ and $\gamma$ correlate as follows:

$$
k=\gamma \rho c_{t}
$$

The coefficient $k$ was determined based on surface energy, when a drop of liquid slides over an inclined plane 8 However, when the fluid flows in the tube near the surface, the adhesive forces will be influenced by the pressure gradient, so it can be assumed that they will change depending on the fluid velocity in the vicinity of the wall.

To explain the effect of the flowing liquid on the value of the adhesion forces in the turbulent flow, let us proceed from a simple example of liquid flow between two parallel walls, as shown in Fig. 2.

By the integration of the centered Navier-Stokes equations for steady flow between two parallel plates a relationship can be derived for shear stresses at a selected point of the surface induced by the effects of adhesive forces:

$$
\tau(0)+\tau(H)=-H \operatorname{grad} p
$$


It is clear from expression (4) that the shear stress at one point on the boundary will depend on the pressure gradient, so it can be assumed that the pressure gradient value here will affect the value of adhesive forces. We suppose that the surface $y=0$ is hydrophobic and the surface $y=H$ is hydrophilic.

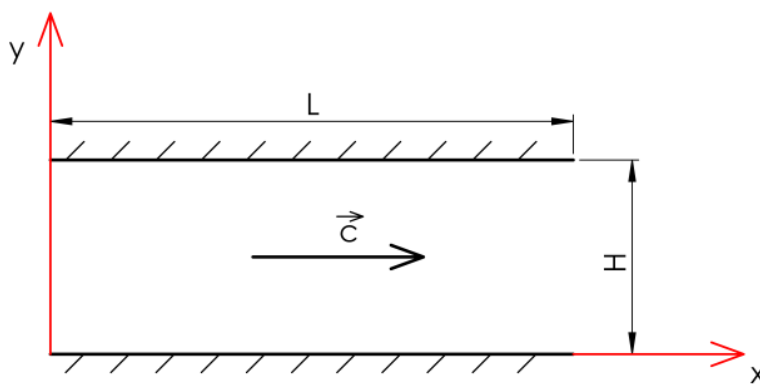

Fig. 2. Coordination system

Therefore, the stresses on the surfaces $y=0$ and $y=$ $H$ will qualitatively vary depending on the turbulent velocity fluctuations. Turbulent fluctuations can be described by different models. Let us speak about the $K-\varepsilon$ model 9,4, where $K$ and $\varepsilon$ are defined by the relations:

$$
\begin{gathered}
K=\frac{1}{T} \int_{t-\frac{T}{2}}^{t+\frac{T}{2}} c_{i}^{\prime} c_{i}^{\prime} d \tau \\
\mathcal{E}=v \frac{1}{T} \int_{t-\frac{T}{2}}^{t+\frac{T}{2}} \frac{\partial c_{i}^{\prime}}{\partial x_{j}} \frac{\partial c_{i}^{\prime}}{\partial x_{j}} d \tau
\end{gathered}
$$

Based on $K$ and $\varepsilon$, the turbulent viscosity $\eta_{T}$ is determined, for which holds:

$$
\eta_{T}=\rho C \mu \frac{K^{2}}{\varepsilon}
$$

From the above mentioned definitions, it is understandable that at the hydrophilic surface to which the fluid adheres the fluctuation components of the velocity $c_{i}^{\prime}$ will be zero. Therefore, for $y=H$ :

$$
\begin{gathered}
y=H: \quad c_{i}^{\prime}=0 ; \quad \frac{\partial c_{i}^{\prime}}{\partial x_{j}} \neq 0 \\
K=0 ; \quad \varepsilon \neq 0 ; \quad \eta_{T}=0 \\
\tau(H)=\eta \frac{\partial c(H)}{\partial y}
\end{gathered}
$$

Another situation occurs in the case of the hydrophobic surface $y=0$. If we consider a liquid slip, the following holds:

$$
\begin{array}{lll}
y=0: & c_{i}^{\prime} \neq 0 ; & \frac{\partial c_{i}^{\prime}}{\partial x_{j}} \neq 0 \\
K \neq 0 ; & \varepsilon \neq 0 ; & \eta_{T} \neq 0
\end{array}
$$

$$
\tau(0)=\left(\eta+\eta_{T}\right) \frac{\partial c(0)}{\partial y}
$$

If both surfaces are hydrophobic, equation (4) can be written in the form:

$$
\tau(0)=-\frac{H}{2} \operatorname{grad} p
$$

Hence, the adhesive stress is a linear function of the pressure gradient. After fitting from (10) and considering the assumption that the adhesive force is a linear function of the slip velocity $c_{S}$, it can also be written:

$$
\tau(0)=\left(\eta+\eta_{T}\right) \frac{\partial c(0)}{\partial y}=-\frac{H}{2} \operatorname{grad} p=k c_{S}
$$

From the foregoing idea it is important to note that in the boundary condition on the hydrophobic surface in the turbulent flow, it is no longer possible to only consider the molecular viscosity, but the turbulent viscosity $\eta_{T}$ as well.

In order to perform a qualitative analysis of laminar and turbulent flow for hydrophilic and hydrophobic surfaces, it is necessary to express the stress tensor by a single, resilient simplified model. For this case we will unfold from the following power model of turbulence.

For stress tensor:

$$
\begin{gathered}
\sigma_{i j}=-\delta_{i j} p+\tau_{i j} \\
\tau_{i j}=2 \alpha_{n} 2^{\frac{n-1}{2}}\left(c_{i j} c_{i j}\right)^{\frac{n-1}{2}} c_{i j}
\end{gathered}
$$

from (14) for the turbulent viscosity applies:

$$
\eta+\eta_{T}=2 \alpha_{n} 2^{\frac{n-1}{2}}\left(c_{i j} c_{i j}\right)^{\frac{n-1}{2}}=\eta_{s}
$$

$\eta_{s}$ represents the sum of molecular and turbulent viscosities. Based on this model, we are now dealing with the problem of stationary flow of incompressible liquid in a circular cross section tube as is shown in Fig. 3.

In the solution of the turbulent flow by the k-epsilon model, the occurrence of the fluctuation components of velocity on the hydrophobic surface is considered. The fluctuation components of the velocity affect the size of the adhesive forces. This fact must also be taken into account when defining the coefficient of adhesion. We assume that the boundary condition for $\varepsilon$ depending on the velocity gradients will not need to be changed.

By analyzing the simplified model of turbulence, it is possible to assess differences in laminar and turbulent flow with consideration of surface hydrophobicity. When the liquid slips over the surface, non-zero fluctuation velocity components occur in the turbulent flow. These determine the non-zero value of the turbulent kinetic energy $K$. This results in a non-zero value of the turbulent viscosity of the liquid on the surface. In addition, the fluctuation velocity components also influence the value 
of the adhesive forces, so it is necessary to include these in the formulation of new boundary conditions for turbulent flow on the hydrophobic surface 11 .

\section{Velocity profiles for laminar and turbulent flow}

Considering the coordinate system of Fig. 2 and the following assumptions,

$$
\boldsymbol{c}=(0, c, 0), \quad c=c(r)
$$

on the basis of this simplification, considering (14) and by the integration of the Navier-Stokes equation, we obtain the relationship for the velocity gradient:

$$
\begin{gathered}
\frac{\partial c}{\partial r}=-\left(\frac{p_{1}-p_{2}}{2 L \alpha_{n}}\right)^{\frac{1}{n}} r^{\frac{1}{n}} \\
\Delta p=p_{1}-P_{2}
\end{gathered}
$$

When we mark

$$
Z_{n}=\left(\frac{\Delta p}{2 L \alpha_{n}}\right)^{\frac{1}{n}}
$$

By the solution of equations (17) we obtain the relation for velocity component $c$ :

$$
c=A-Z_{n} \frac{n}{n+1} r^{\frac{n+1}{n}}
$$

We define the integration constant $A$ from the condition on the boundary of the hydrophobic surface, for which, considering (13), holds:

$$
\begin{array}{r}
r=R: \eta_{s} \frac{\partial c}{\partial r}=-k c \\
\frac{\partial c}{\partial r}=-Z r^{\frac{n+1}{n}} \\
r=R: \frac{\partial c(R)}{\partial r}=-Z R^{\frac{1}{n}}
\end{array}
$$
holds:

From the combination of equations (19) - (21) for $A$

$$
A=\left(\frac{\eta_{s}}{k} Z_{n} R^{\frac{1}{n}}\right)+Z \frac{n}{n+1} R^{\frac{n+1}{n}}
$$

When the surface is hydrophilic

$$
\alpha_{n}=\eta
$$

If the surface is hydrophobic

$$
\alpha_{n}=\eta_{s}\left|\frac{\partial c_{2}(R)}{\partial r}\right|^{1-n}
$$

Considering these assumptions, it holds:

$$
c=\Delta p \frac{R}{2 L}\left\{\frac{1}{k}+\frac{n}{n+1} \frac{R}{\eta_{s}}\left[1-\left(\frac{r}{R}\right)^{\frac{n+1}{n}}\right]\right\}
$$

For the average velocity, it can be written:

$$
c_{S T}=\frac{\Delta p}{2} \frac{R}{L}\left[\frac{1}{k}+\frac{n}{3 n+1} \frac{R}{\eta_{s}}\right]
$$

The velocity on the hydrophobic surface:

$$
c_{s}=\Delta p \frac{R}{2 L} \frac{1}{k}
$$

The following conclusions follow from this solution. The relationship (25) is general and applies to both laminar and turbulent flows. For laminar flow $n=1$, so it will be for:

Hydrophobic surface - laminar flow

$$
\begin{gathered}
c=\Delta p \frac{R}{2 L}\left\{\frac{1}{k}+\frac{1}{2} \frac{R}{\eta}\left[1-\left(\frac{r}{R}\right)^{2}\right]\right\} \\
c_{s}=\Delta p \frac{R}{2 L} \frac{1}{k}
\end{gathered}
$$

Hydrophilic surface - laminar flow

$$
c=\Delta p \frac{R^{2}}{4 L} \frac{1}{\eta}\left[1-\left(\frac{r}{R}\right)^{2}\right]
$$

\section{Hydrophobic surface - turbulent flow}

The $n$ value depends on the Reynolds number, $n<1$

$$
\begin{gathered}
c=\Delta p \frac{R}{2 L}\left\{\frac{1}{k}+\frac{n}{n+1} \frac{R}{\eta_{s}}\left[1-\left(\frac{r}{R}\right)^{\frac{n+1}{n}}\right]\right\} \\
\Delta p=2 \frac{L}{R^{2}} \frac{(3 n+1)}{\frac{1}{k R} \frac{3 n+1}{n}+\frac{1}{\eta_{s}}} \frac{c_{S T}}{n}
\end{gathered}
$$

\section{Hydrophilic surface - turbulent flow}

$$
\begin{gathered}
c=\Delta p \frac{R}{2 L} \frac{n}{n+1} \frac{R}{\eta}\left[1-\left(\frac{r}{R}\right)^{\frac{n+1}{n}}\right] \\
\Delta p=2 \frac{L}{R^{2}} \eta(3 n+1) \frac{c_{S T}}{n} \\
c_{S}=0
\end{gathered}
$$

From the above mentioned results, the following statements arise:

- The sliding velocity in both laminar and turbulent flows is formally the same. Compare (28) and (33). 
- From relation (32), it is obvious that the coefficient of adhesion $k$ and the viscosity on the surface of the pipe $\eta_{s}=\eta+\eta_{T}$ have qualitatively the same effect on the differential pressure. For constant $k$ an increase of $\eta_{s}$ will lead to an increase of the differential pressure.

- It follows from relation (31) that at low values $k$ there will be high values of steady velocity on and near the surface. However, these values generate high fluctuation velocities $c_{i}^{\prime}$, and increase $K$ and $\eta_{T}$. This, however, causes the velocity to decrease both near the surface and on the surface itself, so that also $k$ will be inversely affected. However, this does not affect the mathematical model because constant $k$ and $\eta_{T}$ are considered.

Therefore, the model boundary condition (20) must be modified so that $k$ and also $\eta_{T}$ is dependent on $n$. For the $K-\varepsilon$ model, it will be necessary to adjust the boundary condition for $K$ at the hydrophobic surface. Since now the boundary condition for the $K$ relation is in the form of

$$
K=0
$$

\section{The specification of boundary conditions}

The value of the kinetic turbulent energy density $K(36)$ on the hydrophobic surface will depend on the velocity slip rate. In this sense, it is necessary to formulate a new boundary condition.

\section{$4.1 K-\varepsilon$ turbulence model}

Due to the occurrence of the fluctuation components of velocity $c_{i}^{\prime}$ on the hydrophobic surface and assuming that the fluctuation components of velocity also affect the size of the adhesive forces, it can be assumed:

$$
K=\beta c_{t} c
$$

where $\beta$ is a dimensionless constant, and $c$ is the value of velocity slip of the fluid on the hydrophobic surface, in the tangent direction to the surface. The turbulent viscosity can be determined from definition (3).

The fluctuation velocity components of the liquid on the hydrophobic surface will affect the size of the adhesive forces. Assuming that, it must be taken into account when defining the coefficient of adhesion:

$$
k=\rho c_{t}(\alpha+\beta)
$$

We assume that the boundary condition for $\varepsilon$, depending on the velocity gradients, will not need to be changed.

\subsection{Simplified turbulence model for a tube of circular cross section}

The effect of the fluctuation components of velocity will depend on the Reynolds number and will influence the value of the adhesive forces characterized by the adhesion coefficient $k$ 12. The value of the exponent $n$ is dependent on the Reynolds number. Therefore, for $k$ the following relationship can be proposed, expressing the interaction of the adhesive forces and the force effect of the fluctuation velocity components represented by the kinetic energy of turbulence $K$.

$$
k=\rho c_{t}\left(\alpha+\beta \frac{1-n}{n}\right)
$$

According to the simplified model (11) applied to the liquid flow in a circular cross-section tube, the relationship for turbulent viscosity can be derived. On the basis of the definition of the mixing length $l$, we can say:

$$
\mathrm{l}=\psi(1-n) r, \eta_{T}=-\rho l^{2} \frac{\partial c}{\partial r}
$$

From here and from (31) we get an expression for the resulting viscosity of the liquid on the hydrophobic surface:

$$
\eta_{s}=\frac{\eta}{2}+\frac{1}{2} \sqrt{\eta^{2}+4 \rho\left(\frac{\Delta p}{2 L}\right) \psi^{2}(1-n)^{2} R^{3}}
$$

The new boundary condition expressing the slip of the velocity on the hydrophobic surface will take the form:

$$
\eta_{s} \frac{\partial c}{\partial r}=\rho c_{t}\left(\alpha+\beta \frac{1-n}{n}\right) c
$$

$\alpha, \beta, \Upsilon$ are dimensionless constants. The relationship for the velocity profile (31) will be as follows:

$$
\begin{gathered}
c=\frac{\Delta p}{2} \frac{R}{L}\left\{\begin{array}{c}
\frac{n}{\rho c_{t}[\alpha n+\beta(1-n)]}+ \\
+\frac{n R}{\eta_{s}(n+1)}\left[1-\left(\frac{r}{R}\right)^{\frac{n+1}{n}}\right]
\end{array}\right\} \\
c_{S T}=\frac{\Delta p}{2} \frac{R}{L}\left[\begin{array}{c}
\rho c_{t}[\alpha n+\beta(1-n)] \\
+\frac{n}{3 n+1} \frac{R}{\eta_{s}}
\end{array}\right] \\
c_{S}=\frac{\Delta p}{2} \frac{R}{L}\left[\frac{n}{\rho c_{t}[\alpha n+\beta(1-n)]}\right]
\end{gathered}
$$

From expression (43), which represents the liquid slip on the hydrophobic surface, the influence of turbulent 


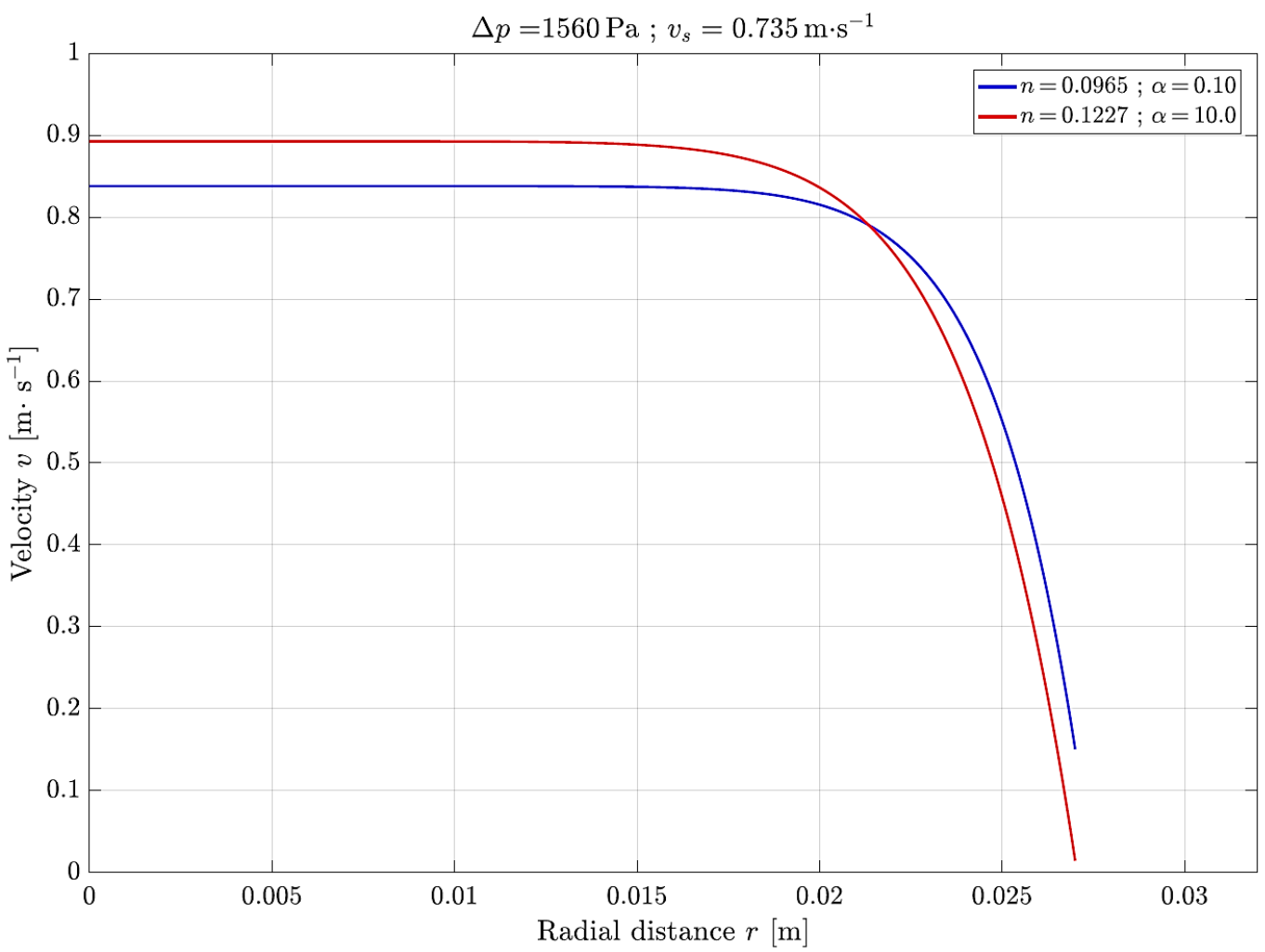

Fig. 3. Turbulent velocity profiles for a constant pressure drop and a constant mean velocity. $\beta=0,1$

fluctuations on the adhesive forces effect is already evident. Figure 3 shows velocity profiles for different adhesion coefficient values. From this, it is clear that the simplified turbulence model for a circular cross-section tube is a good illustration of the shape of the turbulent velocity profile. Note that $\Delta p, c_{S T}$ corresponds to an experiment carried out on a $4 \mathrm{~m}$ length tube.

The new boundary conditions for turbulent flow on the hydrophobic surface can be for the turbulence model $(K$ c) written in the form:

$$
\begin{gathered}
c=0: \quad|\tau| \leq|\tau|_{K} \\
c \neq 0: \tau=\rho c_{t}(\alpha+\beta) c \\
K=\beta c_{t} c
\end{gathered}
$$

\section{Conclusion}

By analyzing the simplified model of turbulence, it was possible to assess differences in laminar and turbulent flow with consideration of surface hydrophobicity. If there is a fluid slip on the surface, a non-zero fluctuation component of the velocity occurs in the turbulent flow. These are determining the non-zero value of the turbulent kinetic energy $K$. This results in a non-zero value of the turbulent viscosity of the liquid on the surface. In addition, the fluctuation components of velocity influence the value of the adhesive forces, so it is necessary to include these facts in the formulation of new boundary conditions (44), (45) for turbulent flow on the hydrophobic surface.
Grant Agency of the Czech Republic, within the projects GA101/15-06621S, GA101/17-19444S is gratefully acknowledged for support of this work.

\section{References}

1. Navier C. L. M. H., "Memoire Surles du Movement des.” Mem Acad. Sci. Inst. France, vol. 1, No. 6, pp. 414-416. (1823)

2. Grunceli BRK, Sandham ND and McHale G. Simulation of laminar flow past a superhydrophobic sqhere with drag reduction and separation delay. Phys Fluids 2013; 25: 043601.

3. Thomas Young: An Essay on the Cohesion of Fluids. Philosophical Transactions of the Royal Society of London. Vol. 95 (1805), 65-87.

4. Vakarelski I. U., Chan D. Y. C., Marston J. O., Thoroddsen S. T.: Dynamic Air Layer on Textured Superhydrophobic Surfaces, Langmuir, 2013, 29 (35), pp 11074-11081

5. Jašíková, D., Němcová, L., Kopecký V., The Methodic for Study of Smart Surfaces Using PIV Technique. AIP Conf. Proc. vol. 80, nr. 8. pp. $80-$ 87 (2014) 
6. Fialová, S.; Pochylý, F.; Kotek, M; Jašíková, D.: Velocity profiles of fluid flow close to a hydrophobic surface, EPJ Web of Conferences 143, 02023 (2017) DOI: $10.1051 /$ epjconf/201714302023

7. Fialová, S., Pochylý, F., Havlásek, M., Malík, J.: Influence of boundary conditions on fluid flow on hydrophobic surfaces, AIP Conf. Proc - 36th Meeting of Departments of Fluid Mechanics and Thermodynamics (2017)

8. Fialová, S.; Pochylý, F.: Identification and Experimental verification of the adhesive coefficient of hydrophobic materials, Wasserwirtschaft Extra, 1/2015, ISSN 0043 0978, pp. 125-129

9. Mohammadi, B., \& Pironneau, O. (1993). Analysis of the K-epsilon turbulence model. France: Editions MASSON.
10. Mansour, N. N.; Kim, J.; Moin, P. Near-wall kepsilon turbulence modeling, 6th Symposium on Turbulent Shear Flows, France, Proceedings (A8838951 15-34). University Park, PA, Pennsylvania (1987), p. 17-4-1 to 17-4-6.

11. Kučera, R.; Šátek, V.; Haslinger, J.; Fialová, S.; Pochylý, F. Modelling of Hydrophobic Surfaces by the Stokes Problem with the Stick- Slip Boundary Conditions. Journal of Fluids EngineeringTransactions of the ASME, 2017, vol. 139, no. 1, p. 0112021-0112029. ISSN: 0098-2202.

12. Volkov AV, Parygin AG, Lukin MV, et al. Analysis of the effect of hydrophobic properties of surfaces in the flow part of centrifugal pumps on their operational performance. Therm Eng 2015; 62: 817824. 